\title{
Design of Novel Low-Power and High-Efficiency Class-d Audio Amplifier
}

\author{
Mohammad Mahdi Hajhashemkhani ${ }^{* 1}$, Abdalhossein Rezai ${ }^{2}$ and Ahmad Karimi ${ }^{3}$ \\ ${ }^{1,2,3}$ ACECR institute of higher education, Isfahan branch, Isfahan 84175-443, \\ Iran \\ 1'Mm_hhkh@gmail.com, 2 rezaie@acecr.ac.ir, ${ }^{3}$ Ahmadkarimi260@gmail.com
}

\begin{abstract}
This paper presents a high-efficiency class-D audio amplifier. In the proposed architecture for the class-D amplifier, all stages of class-D amplifier are designed carefully. A second order delta sigma modulation, high switching frequency and three passive feedbacks are used in modulation stage. A special filter is designed to improve the quality of the proposed class-D audio amplifier. The proposed class-D amplifier architecture is simulated using MATLAB. The simulation results show that the proposed class-D amplifier architecture has low THD (<0.001), High SNR $(>80 d B)$, and high efficiency (>95\%), which demonstrate that the proposed class-D amplifier architecture have the best performance in terms of THD, and efficiency in comparison with other class-D amplifier architectures.
\end{abstract}

Keywords: Class-D audio amplifier, THD, SNR, Efficiency, Power amplifier

\section{Introduction}

In the last decades, high power audio amplifiers have been widely used in our life [1]. The goal of amplifiers is to reproduce the input signal with the desired power and quality in the output [1]. Audio amplifiers can be categorized in 5 groups: (1) class-A amplifiers, that consist of a simple Bipolar Junction Transistor (BJT), which is shown in Figure 1, (2) class-B amplifiers, (3) class-AB amplifiers, (4) class-C amplifiers, and (5) class-D amplifiers [2].

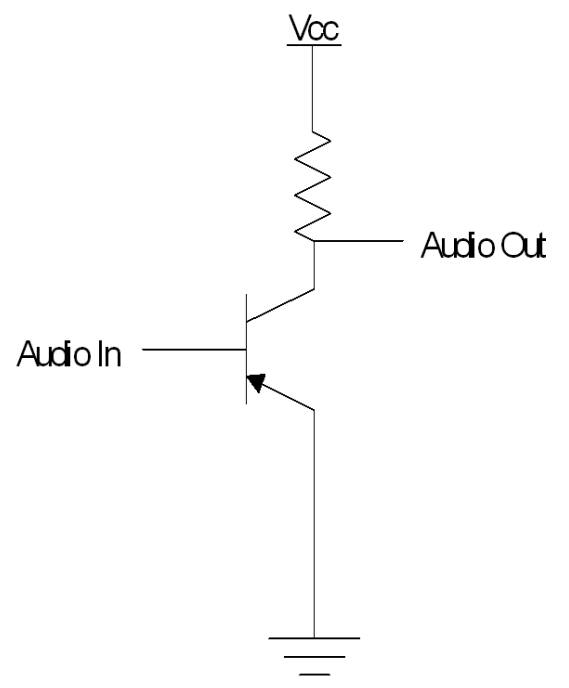

Figure 1. Class-A Amplifier [2]

*corresponding author 
In class-A amplifier, the input signal is applied to the base of BJT and the output signal is driven out from its collector. It should be noted that the efficiency of class-A amplifier is less than $50 \%[1,3]$.

To improve the quality of amplifier, several system configurations have been utilized such as class-A, class-B or class-AB amplifiers, but the efficiency of these linear amplifiers is less than $78 \%$ [2]. To achieve higher efficiency and improve the quality of amplifiers, the class-D configuration can be utilized [4]. The class-D configuration contains three main stages: (1) modulation stage, (2) power stage, and (3) filtering stage [4].

The utilized method in the modulation stage can be divided into two categories: (1) Pulse Width Modulation (PWM) [3], and (2) Pulse Density Modulation (PDM) such as delta sigma modulator [4]. In the PWM modulation, the comparator generates a pulse signal based on the input signal and a carrier signal, but this method leads to unwanted signals and harmonics due to carrier signal's low frequency [5].

On the other hand, the PDM method is utilized to design low power amplifiers. However, unwanted harmonic in this method is lower than the PWM method [6]. Several methods and structures have been proposed to increase the efficiency of class-D amplifiers based on its stages [7-21].

In this paper, a new architecture is presented and evaluated for the PDM class-D audio amplifier. In the modulation stage of the proposed class-D audio amplifier, a second order delta sigma modulator with three state feedbacks is utilized. The power stage configuration and component are designed very carefully. The filtering stage is designed to improve the output quality with respect to the power stage output. The proposed architecture for the class-D audio amplifier was simulated using MATLAB. Our simulation results show that the proposed class-D audio amplifier has several advantages compared to other class-D audio amplifiers in [17 - 21]

The remainder of this paper is organized as follows: in Section 2, a background of the class-D amplifier is discussed. In Section 3, the proposed method is discussed and simulated. Section 4 compares the proposed class-D audio amplifier with other class-D amplifiers. Finally, Section 5 concludes this paper

\section{Background}

\subsection{Class-D amplifier}

A simple PDM class-D audio amplifier block diagram is shown in Figure 2 [7, 9].

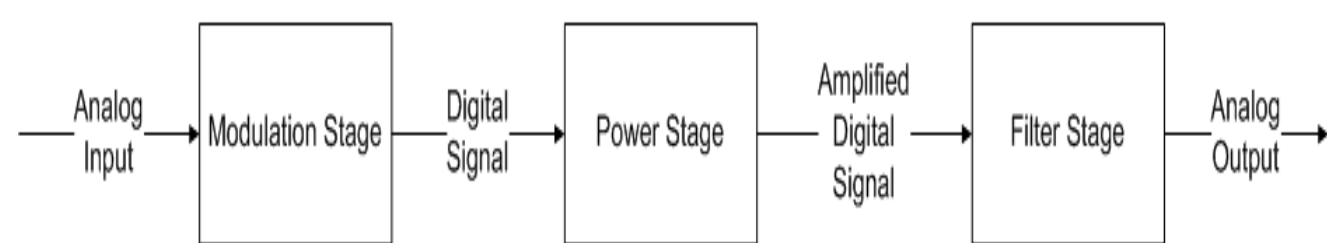

Figure 2. A Simple PDM Class-D Audio Amplifier Block Diagram [7, 9]

As it is shown in Figure 2, this type of amplifier has multiple stages. Note that to have better output and improve the efficiency of this amplifier, these stages should be optimized. A simple class-D audio amplifier with an LC filter is shown in Figure 3 [8]. 


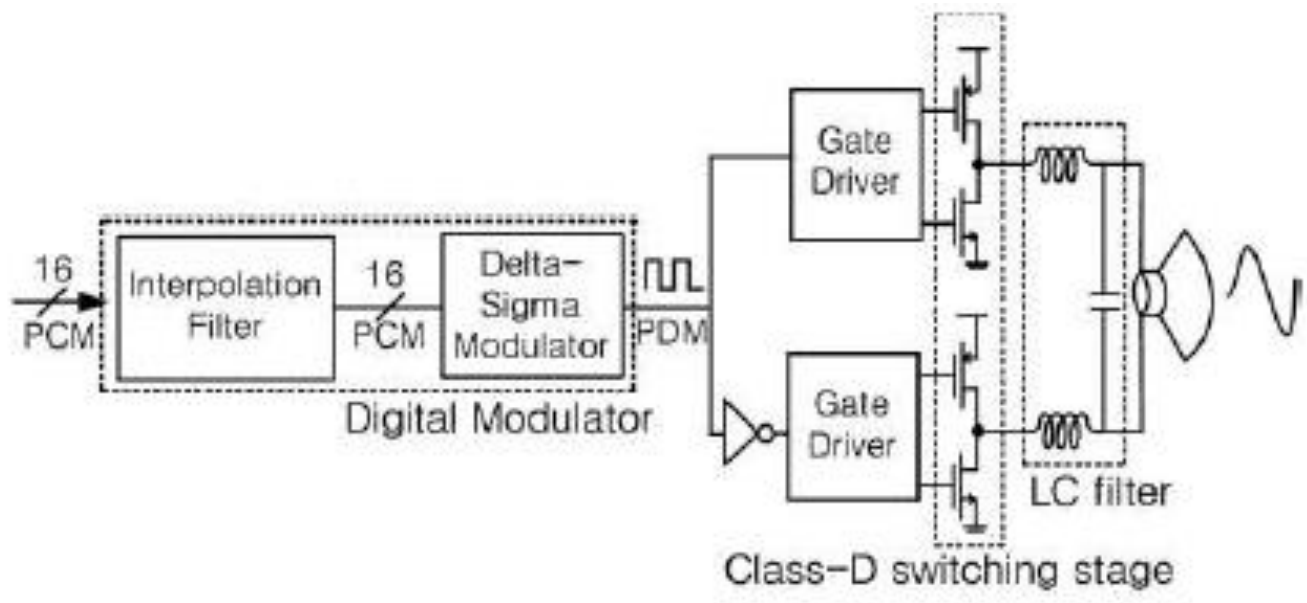

Figure 3. A Simple Class-D Audio Amplifier Using LC Filter [9]

As it is shown in Figure 3, the input analog signal is first modulated and converted to a digital signal. Then, the modulated signal is applied to the gate of Metal Oxide Field Effect Transistors (MOSFETs). The power stage, class-D switching stage, is responsible for increasing the power of achieved signal [10]. The most important factor for these MOSFETs is their triode region. This factor must be decreased as much as possible [11].

\subsection{PDM Method}

The first order delta sigma modulation with one state feedback block diagram is shown in Figure 4 [14].

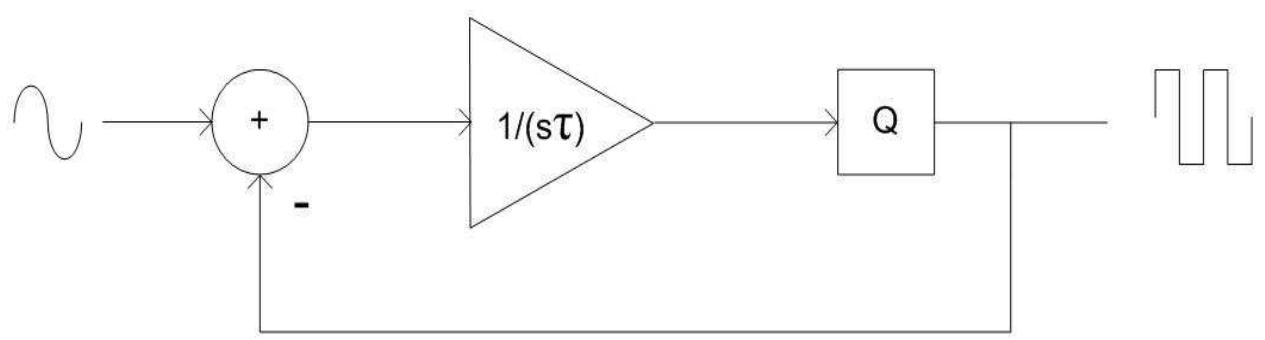

Figure 4. First Order Delta Sigma Modulation Block Diagram [14]

Figure 5 shows the utilized circuit for the first order delta sigma modulation. This circuit is simulated using Proteus. 


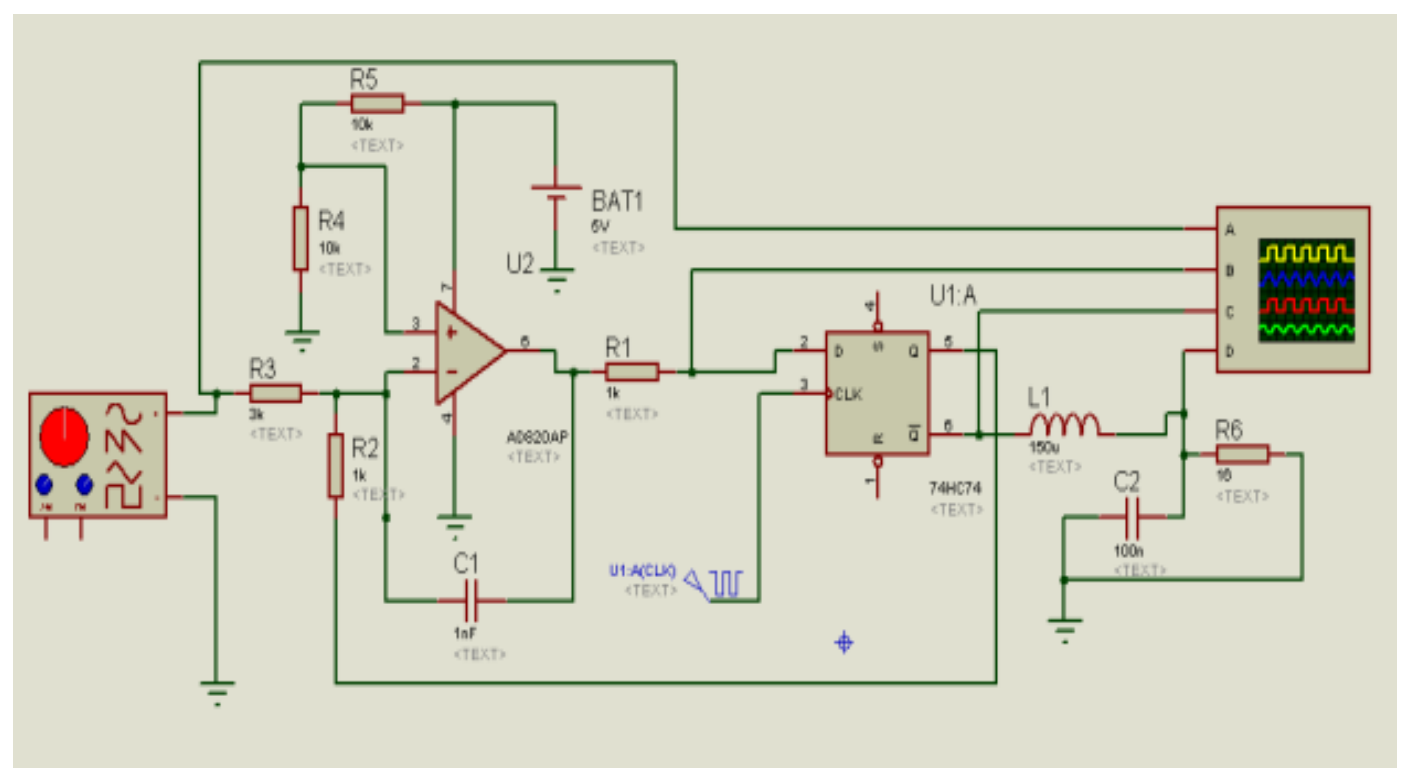

Figure 5. First Order Delta Sigma Modulation with One State Feedback

The results of this simulation are shown in Figure 6.

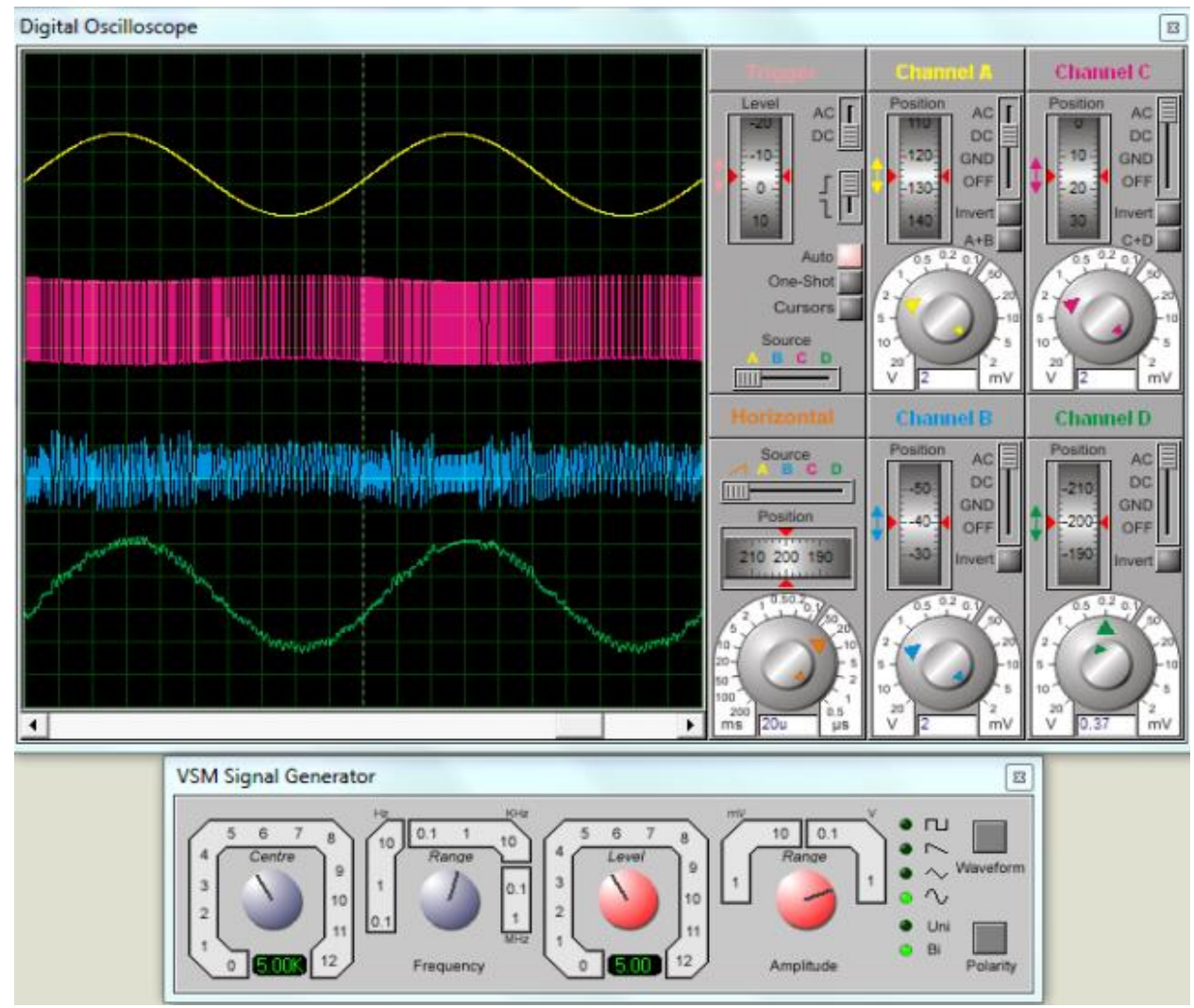

Figure 6. Result of First Order Delta Sigma Modulation with One State Feedback

It should be noted that the quantize complexity and non-linearity can be the source of some disturbance in the output. To simulate a first order delta sigma modulation with consideration of these unwanted signals, a simple noise signal is added as an input to this system. This system formula is shown as follows [14]: 


$$
V_{\text {OUT }}=\left[\frac{1}{1+s \tau}\right] V_{W N}+\left[\frac{s \tau}{1+s \tau}\right] \epsilon
$$

To gain a perfect output, it is needed to push the noise far from audio band and filter it at filtering stage [15]. This equation shows that by finding the perfect frequency for modulation, it may be possible to filter this unwanted noise.

\subsection{The Power Stage}

There are two configurations for power stage in class-D amplifiers: (1) half-bridge configuration, and (2) full-bridge configuration. In both of these configurations, the main purpose is done by MOSFETs and their drivers. To improve this stage performance, the used MOSFETs must be chosen carefully and for high efficiency purpose, some of new designed MOSFETs are used and their results are compared to achieve the best possible output. Full-bridge configuration has more components compared to half-bridge configuration, but in audio amplifiers, it is needed to cancel DC offsets and harmonic distortion. This problem can be solved only in full-bridge configuration. So, the fullbridge configuration is suitable for class-D amplifier. Figure 7 shows a simple full-bridge configuration [16].

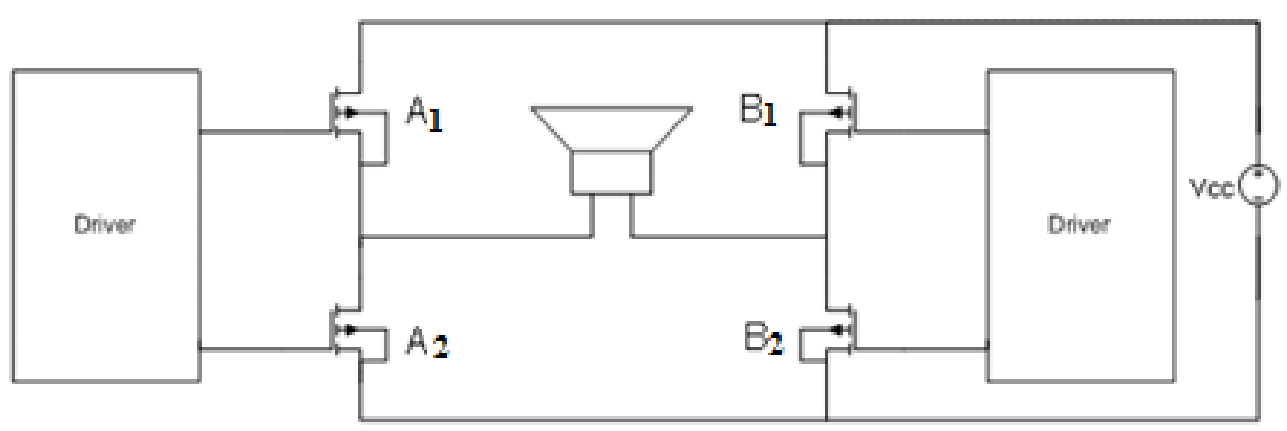

Figure 7. A Simple Full-Bridge Configuration [16]

As it is shown in Figure 7, in full-bridge configuration, two MOSFETs are in on-state, for example $A_{1}$ and $B_{2}$, and the other MOSFETs are in off-state, $A_{2}$ and $B_{1}$ [15]. The most important rule to follow is that, $\mathrm{A} 1$ and $\mathrm{A} 2$ or $\mathrm{B} 1$ and $\mathrm{B} 2$ should never be in on-state at the same time. To control the MOSFETs, a circuit called MOSFET's driver is needed. This circuit must be designed based on the MOSFET's characteristics such as MOSFET's gate capacitance $\left(\mathrm{C}_{\mathrm{g}}\right)$ or Drain Source resistance $\left(\mathrm{r}_{\mathrm{ds}}\right)$ [16].

\subsection{Filter Stage}

At this stage any unwanted noise or signals must be filtered before they reach the output. After the input signal is modulated and amplified, it would be still in digital form and speaker's input must be an analog signal [17]. Before choosing a suitable filter, it is important to evaluate system's output. To test and evaluate a system output's quality, three factors must be calculated: (1) Signal to Noise Ratio (SNR), (2) Spurious Free Dynamic Range (SFDR) and (3) Total Harmonic Distortion (THD) [6].

SNR is used to determine the power of signal in relation to noise's power and it is formulated as follows [6]:

$$
S N R=10 \log \frac{P_{\text {gignal }}}{P_{\text {noise }}}
$$

SNR measures the noise power in the background, which is in audio range, and compares it with the main signal [6]. 
SFDR is a very important parameter, which is used when an Analog to Digital Converter (ADC) is used in the system [18]. This evaluation is used to determine the ratio between desired signal and any unwanted signals, which may be the results of harmonics or system complexity [19]. Sometime SFDR is the result of a harmonic, but to evaluate the ratio between all of the harmonics and output another parameter is used, which is called THD [15, 17]. This two parameters depend on modulation technique and the complexity of system.

Filters are divided into two categories: (1) passive filter and (2) active filter. Passive filters contains only passive component such as resistors, inductors and capacitors, where active filters contains components such as operational amplifiers.

\section{The Proposed Model}

In this paper, we proposed a novel class-D audio amplifier. In the proposed class-D audio simplifier, all amplifier stages are carefully designed. This section presents the developed architectures for all stages of the proposed class-D audio amplifier.

\subsection{Modulation Stage}

Figure 8 shows the modulation stage in the proposed class-D audio amplifier.

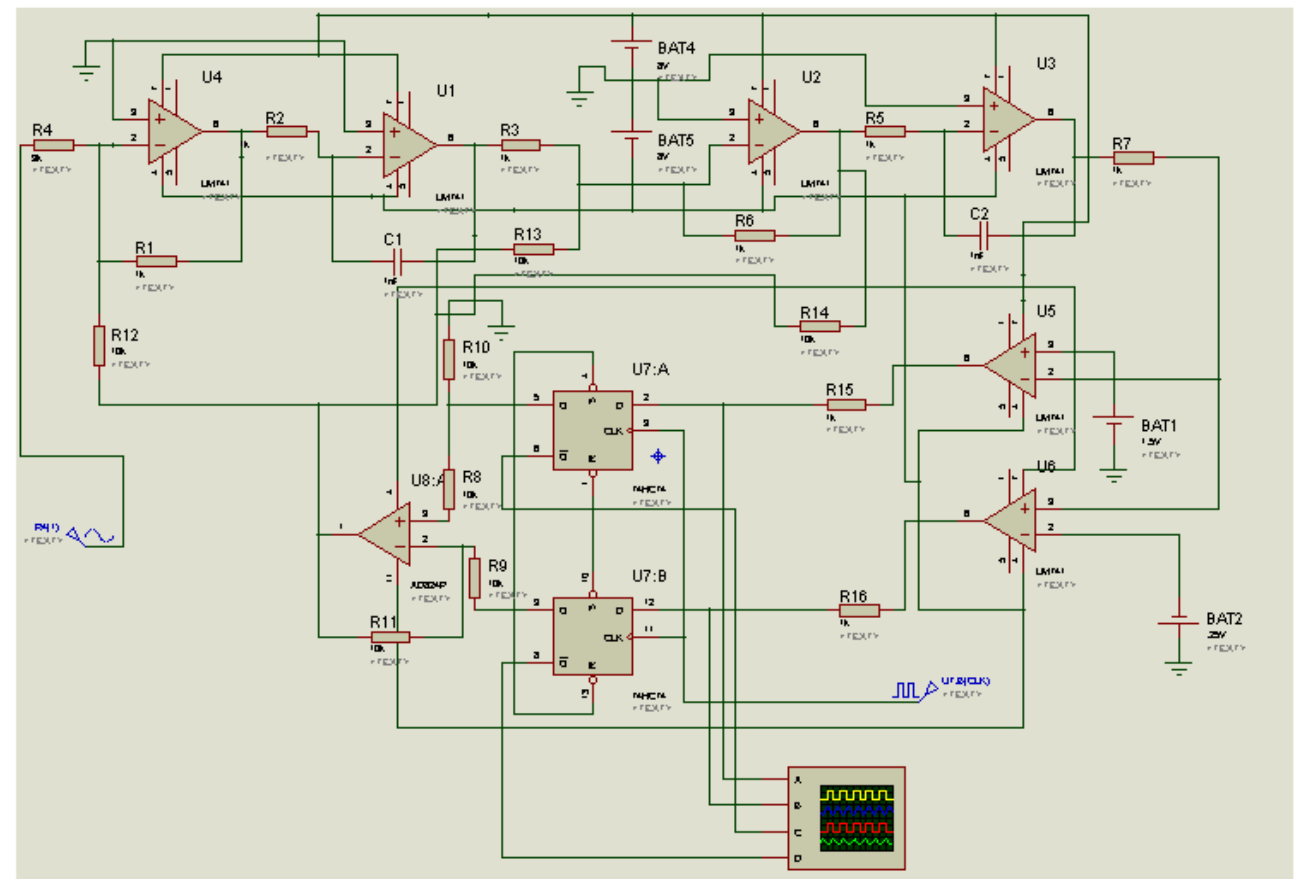

Figure 8. The Utilized Circuit for the Modulation Stage of the Proposed Class-D Audio Amplifier

As it is shown in Figure 8, an integrator is added to the first order delta sigma modulator. Therefore, it is changed into a second order delta sigma modulator. So, this new system can have two or three feedbacks to have a steady state output. The output is improved slightly, but it is not enough; however, when the value of three feedback resistors is changed, a steady state signal with a good quality is achieved in the output. This system model is simulated in MATLAB. Figure 9, shows the utilized model in MATLAB. 


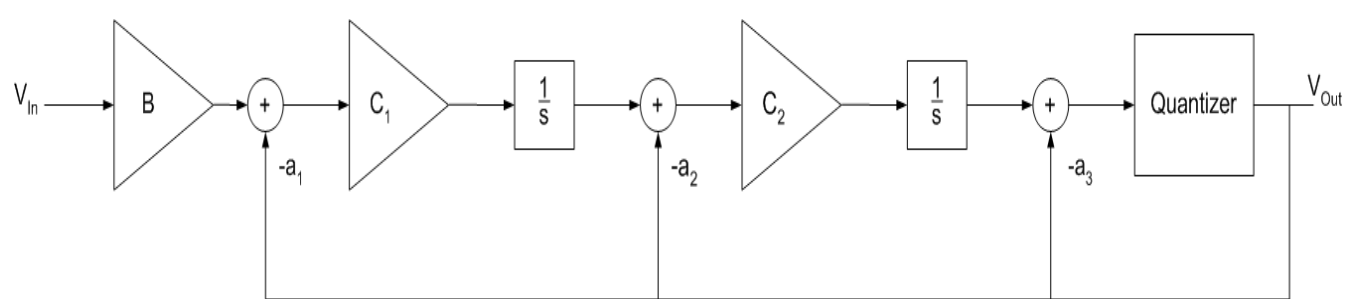

Figure 9. Delta Sigma Modulation with Three State Feedbacks

By changing each parameter of the modulation stage, the output signal is also changed. Therefore, the modulation output quality varies. To determine the value of each coefficient, all of these parameters are changed in MATLAB to have a steady state system with a good quality output signal. It should be noted that these values can be changed if the following term's value remains the same $-a_{3} \cdot s^{2},-a_{2} \cdot C_{2} \cdot s,-a_{1} \cdot C_{1} \cdot C_{2}$ and B.C1.C2. This stage output is given in Figure 10.

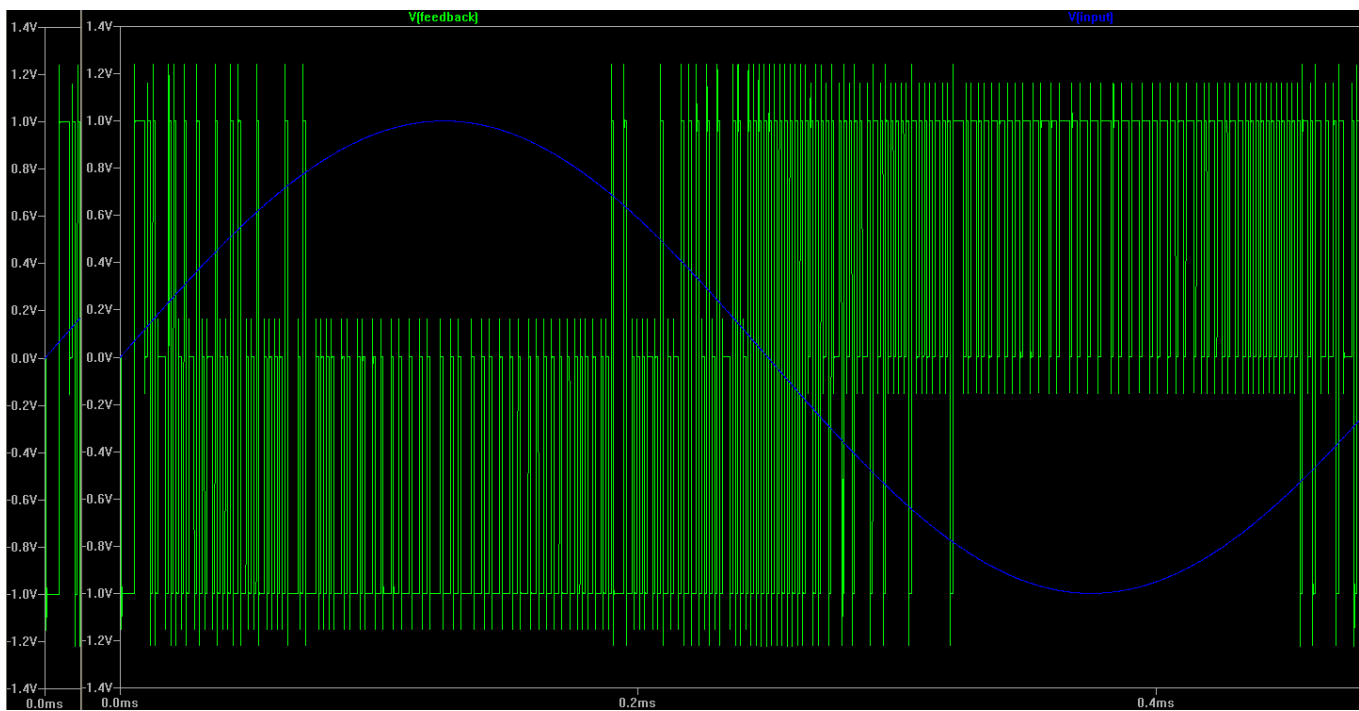

Figure10. Second Order Delta Sigma Modulation Output

The modulator frequency has an important role in the performance of the class-D audio amplifier. If an extremely high frequency is used for modulator, the component might break down, but these high frequencies have never been used, due to system's limitation. To find the best modulation frequency, first the other two stage's configuration must be designed, and then in relation to the output efficiency and quality, each stage's parameters would be determined.

\subsection{Power Stage}

In this paper, we utilized full-bridge configuration to increase the efficiency of the proposed amplifier. Digital signals are applied to the input and the output is monitored. The results show that an inverted form of the input is shown in the output with the amplitude of $45 \mathrm{v}$. This result concluded that this stage works properly.

As it is explained before, a very important part of the power stage is MOSFET's driver. To drive these MOSFETs, a new circuit should be designed. The utilized circuit for the MOSFET driver is shown in Figure 11. 


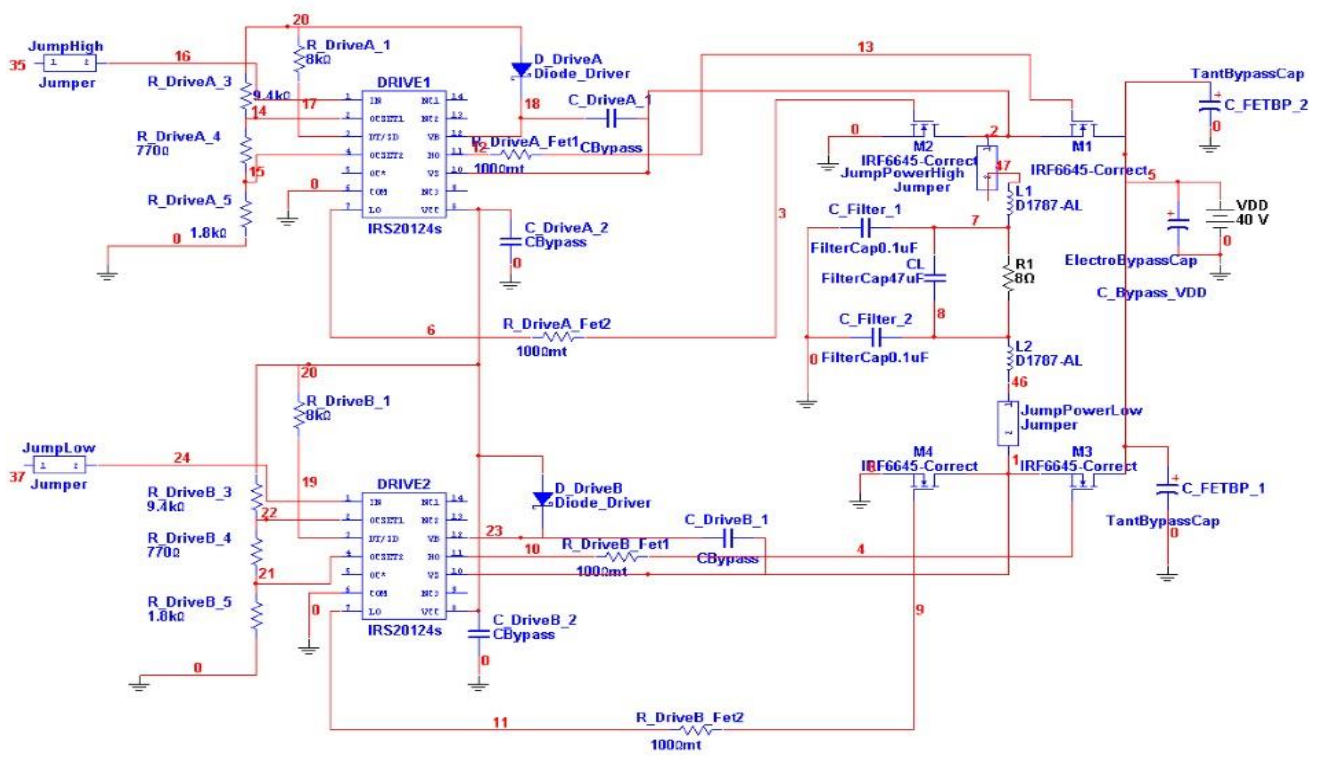

Figure 11. The Utilized Driver for the MOSFETs in the Proposed Class-D Audio Amplifier

\subsection{Filter Stage}

In this paper, we utilized passive filter. The Butterworth filter is an important filter, which is chosen to be used in the proposed class-D audio amplifier. This filter formula is determined using its components and their configurations. The second order half circuit Butterworth filter is shown in Figure 12.

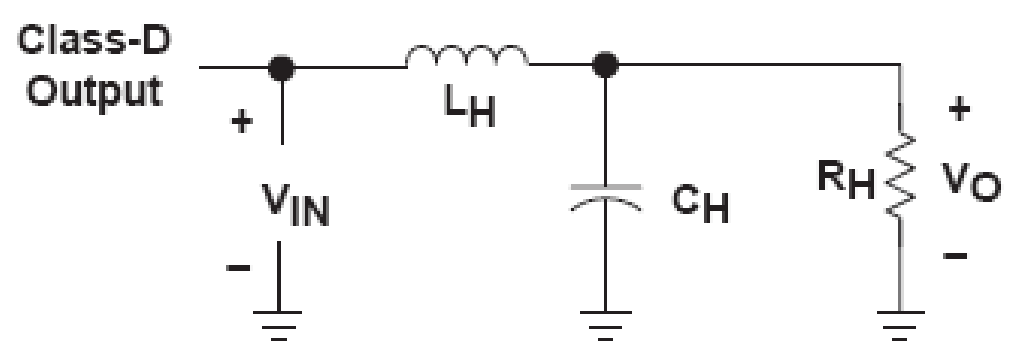

Figure 12. The Butterworth Filter Schematic

This filter formula is as follows

$$
\begin{gathered}
R_{H}=\frac{R_{\mathrm{L}}}{2} \\
C_{H}=\frac{1}{2 \pi f_{G} \sqrt{2} R_{H}} \\
L_{H}=\frac{\sqrt{2} R_{H}}{2 \pi f_{g}}
\end{gathered}
$$

Using these equations, the ideal values for these components are computed. To gain the outmost efficiency, the filter configuration should be changed. Instead of using two capacitances, which are connected to the ground, one capacitor is placed between two half circuits. Moreover, to compensate the other capacitors, the two resistors are replaced with 
capacitors. These changes help to improve the efficiency. The final configuration for this filter is given in Figure 13.

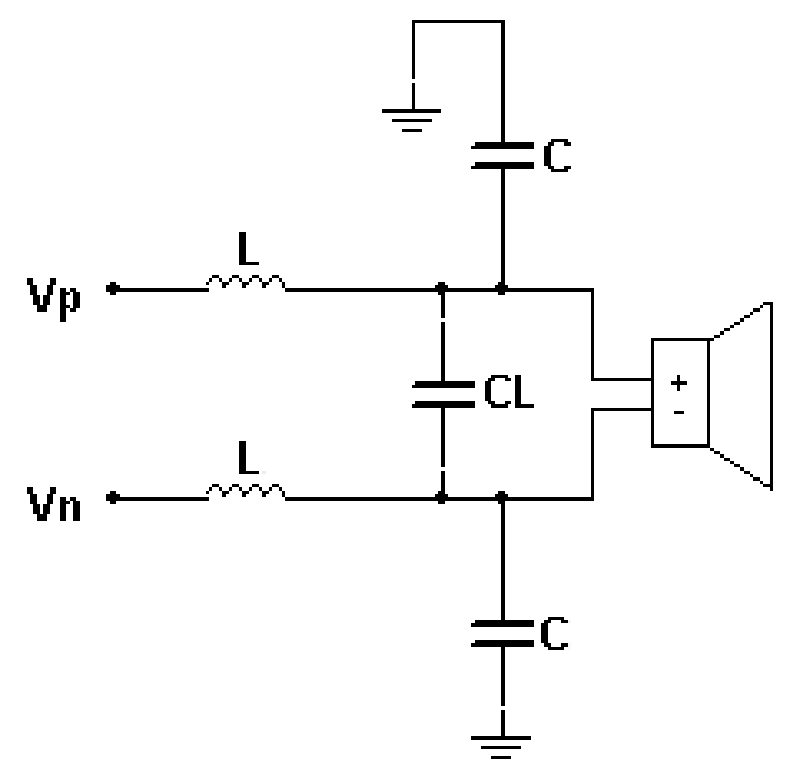

Figure 13. The Utilized Filter in the Proposed Class-D Audio Amplifier

This configuration is final, but the value of these components should be selected in relation to the system's output efficiency.

\subsection{Quality Parameters}

This system quality parameters can be categorized in 4 groups: (1) efficiency, (2) frequency response, (3) THD, and (4) SNR [2, 6]. The first and most important parameter for this system is efficiency, which is computed as follows:

$$
\text { Efficiency }=\frac{P_{\text {out }}}{P_{\text {in }}}
$$

Where:

$$
\begin{gathered}
P_{\text {in }}=V_{\text {in }(D C)} \cdot I_{\text {in }(D C)} \\
P_{\text {out }}=\frac{V_{\text {ount }(R M S)}^{2}}{R_{\text {lond }}}
\end{gathered}
$$

Then

$$
\text { Efficiency }=\frac{V_{\text {in }(D C)} \times I_{\text {in }(D C)}}{\frac{V_{\text {owut }(R M S)}^{2}}{R_{\text {load }}}}
$$

It should be noted that SNR is computed using (2).

The frequency response is formulated as follows:

$$
\text { Gain }=\frac{V_{\text {out (RMS) }}}{V_{\text {in (RMS) }}}
$$

The THD of this system is also computed as follows:

$$
\text { THD }=\frac{\sqrt{h_{1}^{2}+h_{2}^{2}+h_{a}^{2}+h_{4}^{2}+h_{5}^{2}}}{\text { original signal }} .100
$$

To find the perfect MOSFET, the system output is observed and tested by changing the modulator frequency from $1 \mathrm{MHz}$ to $30 \mathrm{MHz}$. For each transistor, there is an ideal frequency, for example: IRF3808 ideal frequency is $2 \mathrm{MHz}$. In this paper we utilized IRS20124S (Pbf) at 5MHz modulation frequency, which leads to a near perfect power 
efficiency (96\%) and low THD. This transistor special characteristic is its low drain source resistance $\left(\mathrm{r}_{\mathrm{ds}}\right)$.

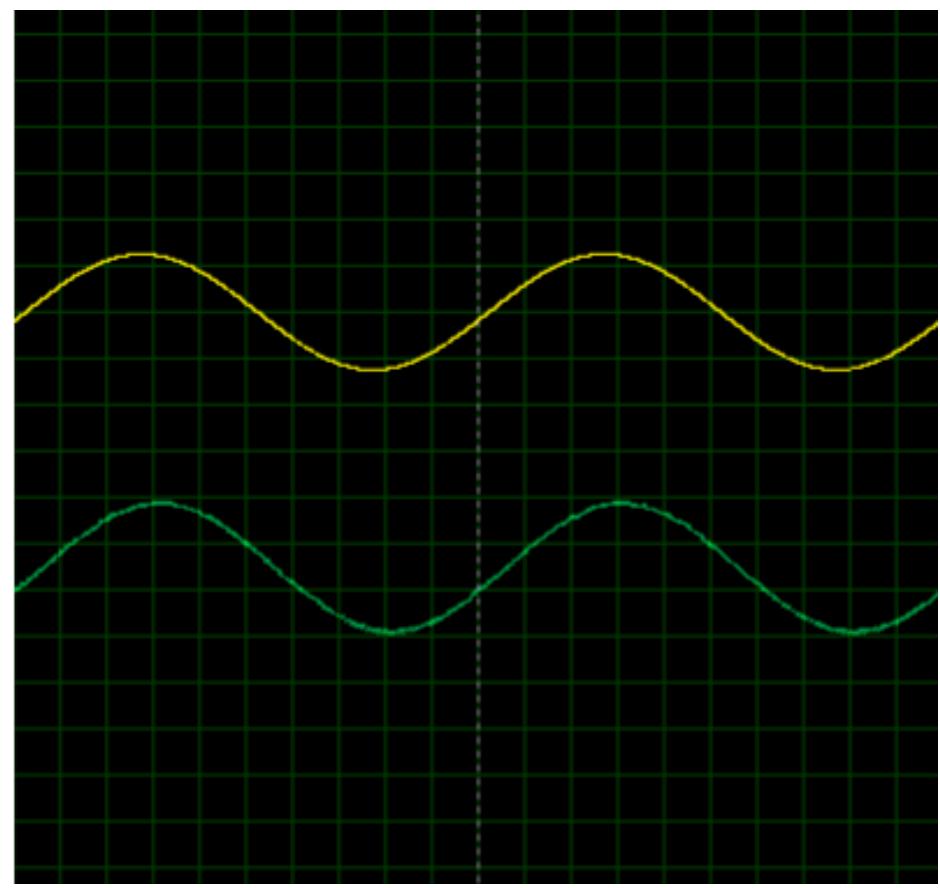

Figure 14. The Simulation Results of the Proposed Class-D Audio Amplifier

The selected values for filter components are $C_{L}=0.47_{\mu F}$ and $C=0.1_{\mu F}$ and $L=20_{\mu H}$. A very important parameter for choosing the inductor is that this component must have a low resistance. It is because all of the system output is passed through the inductors and then into the speakers. So, they must have the lowest possible resistance.

\section{Comparison}

The proposed architecture for the class-D audio amplifier is simulated using MATLAB 2016b. Table 1 summarizes the simulation results of the proposed class-D audio amplifier in comparison with other class-D audio amplifiers in [17-21].

Table 1. The Comparison Table for Class-D Audio Amplifiers

\begin{tabular}{|c|c|c|c|c|c|c|}
\hline Parameters & $\begin{array}{c}\text { This } \\
\text { paper }\end{array}$ & {$[17]$} & {$[18]$} & {$[19]$} & {$[20]$} & {$[21]$} \\
\hline $\begin{array}{c}\text { Modulator } \\
\text { architecture }\end{array}$ & $\begin{array}{c}\text { Digital } \\
\Sigma \Delta\end{array}$ & PWM & PWM & PWM & DSP & $\begin{array}{c}\text { Digital } \\
\Sigma \Delta\end{array}$ \\
\hline VDD & $50 \mathrm{~V}$ & $60 \mathrm{~V}$ & $20 \mathrm{~V}$ & $50 \mathrm{~V}$ & $18 \mathrm{~V}$ & $80 \mathrm{~V}$ \\
\hline P $_{\text {Out max }}$ & $80 \mathrm{~W}$ & $200 \mathrm{~W}$ & $20 \mathrm{~W}$ & $240 \mathrm{~W}$ & $13 \mathrm{~W}$ & $45 \mathrm{~W}$ \\
\hline $\begin{array}{c}\text { Peak SNR } \\
(\mathrm{dB})\end{array}$ & 78 & 82.2 & 91 & 74.1 & 87 & 92.8 \\
\hline $\begin{array}{c}\text { THD+N } \\
\text { Efficiency } \\
(\%)\end{array}$ & $0.001 \%$ & $0.017 \%$ & $0.01 \%$ & $0.1 \%$ & $0.07 \%$ & $0.015 \%$ \\
\hline $\begin{array}{c}\text { Bandwidth } \\
\text { (kHz) }\end{array}$ & 30 & 90 & 89 & N/A & 88 & 93 \\
\hline \multicolumn{2}{c}{} & 20 & 20 & 20 & 14 & 20 \\
\hline
\end{tabular}

Based on our simulation results, which are shown in Table 1, the proposed class-D audio amplifier has an improvement compared to class-D audio amplifier in [17-21] in 
terms of THD, efficiency, and bandwidth. Although, the SNR comparison shows that this parameter is decreased by about $15 \%$ compared to [21], but the output power and bandwidth are increased by about $70 \%$ compared to [21]. Moreover, these results denote that the performance of this system is improved. So, the proposed class-D audio amplifier can be more useful compared to other class-D audio amplifiers.

\section{Conclusion}

Amplifiers play an important role in today's technology. Its main concerns are efficiency, output quality and output power. In this paper, a new architecture for the PDM class-D audio amplifier is designed and simulated. The modulator architecture of the proposed class-D audio amplifier consists of a second order delta sigma modulator with three state feedbacks. The power stage configuration and component are tested and chosen very carefully. The filtering stage is designed to improve the output quality with respect to the power stage output. The proposed architecture for the class-D audio amplifier is simulated using MATLAB. Our simulation results showe that the proposed class-D audio amplifier has several advantages compared to other class-D audio amplifiers in [17 - 21].

\section{References}

[1] X. Jiang, J. Song, D. Cheung, M.-S. Wang, and S. K. Arunachalam, "Integrated Class-D Audio Amplifier With 95\% Efficiency and 105 dB SNR", IEEE Journal of Solid-State Circuit, vol. 49, no. 11, (2014), pp. 2387-2396.

[2] J. Honda and A. Jonathan, "Class D Audio Amplifier Basics," IEEE Solid State Circuits, vol. 35, no. 10, (2014), pp. 119-120.

[3] V. Adrian, J. S. Chang and B. H. Gwee, "A Low Voltage Micro Power Digital Class-D Amplifier Modulator for Hearing Aids", IEEE Transaction on Circuits System, vol. 56, no. 2, (2009), pp. 337 349.

[4] W. C. Wang and Y. H. Lin, "IEEE "A $118 \mathrm{~dB}$ PSRR, 0.00067\% (-103.5 dB) THD+N and 3.1 W Fully Differential Class-D Audio Amplifier With PWM Common Mode Control”, Journal of Solid-State Circuit, vol. 51, no. 12, (2016), pp. $2808-2818$.

[5] B. H. Leung and S. Sutarja, "Multi-bit Sigma-Delta A/D Converter Incorporating a Novel Class of Dynamic Element Matching Techniques," IEEE Transaction on Circuits System, vol. 39, no. 1, (1992), pp. 35-51.

[6] R. Schreier and G. C. Temes, "Understanding Delta-Sigma Data Converters", New York: Wiley-IEEE Press, (2004).

[7] M. T. Tan, J. S. Chang, H. C. Chua and B. H. Gwee, "An Investigation into the Parameters Affecting Total Harmonic Distortion in Low-Voltage Low-Power Class-D Amplifiers", IEEE Transaction on Circuits System, vol. 50, no. 10, (2003), pp. 1304-1315.

[8] K. Nielsen, "Audio Power Amplifier Techniques with Energy Efficient Power Conversion", Ph. D. Thesis, Department of Applied Electronics, Building 451 Technical University of Denmark, (1992).

[9] B. P. Brandt and B. A. Wooley, "A Low-Power, Area-Efficient Digital Filter for Decimation and Interpolation", IEEE Journal of Solid-State Circuits, vol. 29, no. 6, (1994), pp. 679-687.

[10] H. Ma, R. van der Zee and B. Nauta, "Design and Analysis of a High efficiency high-voltage class-D power output stage", IEEE Journal of Solid-State Circuits, vol. 49, no. 7, (2014), pp. 1514-1524.

[11] T. Ge and J. S. Chang, "Modeling and Technique to Improve PSRR and PS-IMD in analog PWM ClassD Amplifiers", IEEE Transaction on Circuits and Systems, vol. 55, no. 6, (2008), pp. 512-516.

[12] H. Vander, R. Zee and B. Nauta, "A High-Voltage Class-D Power Amplifier with Switching Frequency Regulation for Improved High-Efficiency Output Power Range", IEEE Journal of Solid State Circuits, vol. 50, no. 6, (2015), pp. 1451-1462.

[13] K. Svjetlana, T. P. Brianin and J. Gali , "Intermodulation Distortion of Class D Audio Amplifier using Pulse Density Modulation", Zooming Innovation in Consumer Electronics International Conference (ZINC), IEEE, (2016).

[14] K. Kang, J. Roh, Y. Choi, H. Roh, H. Nam and S.Lee, "Class-D Audio Amplifier Using1-bit Fourthorder Delta-Sigma Modulation”, IEEE Transaction on Circuits System, vol. 55, no. 8, (2008), pp. 728-732.

[15] S. H. Yang, Y. H. Yang, K. H. Chen, Y. H. Lin, T. Y. Tsai, J. R. Lin and C. C. Lee, "A Low THD Class-D Audio Amplifier with Dual-Level Dual-Phase Carrier Pulse-Width Modulation", IEEE Transaction on industrial electronics, vol. 62, no. 11, (2015), pp. 7181 - 7190. 
[16] A. I. Hussein, A. N. Mohieldin, F. Hussien and A. Eladawy, "A Low-Distortion High-Efficiency ClassD Audio Amplifier Based on Sliding Mode Control”, IEEE Transaction on Circuit and Systems, vol. 63, no. 8, (2016), pp. 713 - 717.

[17] M. Berkhout, “An Integrated 200-W Class-D Audio Amplifier”, IEEE Journal of Solid State Circuits, vol. 38, no. 7, (2003), pp. 1198-1206.

[18] P. Morrow, E. Gaalaas and O. McCarthy, "A 20-W Stereo class-D Audio Output Power Stage in 0.6- $\mu \mathrm{m}$ BCDMOS Technology", IEEE Journal of Solid-State Circuits, vol. 39, no. 11, (2004), pp. 1948-1958.

[19] F. Nyboe, "A 240W Monolithic Class-D Audio Amplifier Output Stage", ISSCC Digital Technology Papers, (2006); pp. 1346-1355.

[20] J. Liu, "A 100 W 5.1-Channel Digital Class-D Audio Amplifier with Single-Chip Design”, IEEE Journal of Solid-State Circuits, vol. 47, no. 6, (2012), pp. 1344-1354.

[21] H. Ma, R. van der Zee and B. Nauta, "An Integrated 80V 45W Class- D Power Amplifier with OptimalEfficiency-Tracking Switching Frequency Regulation”, IEEE Journal of Solid State Circuits, (2014), pp. 286-288. 